\title{
ASSOCIAÇÃO ENTRE A RESISTÊNCIA INSULÍNICA, SÍNDROME METABÓLICA E FATORES DE RISCO CARDIOVASCULAR ISOLADOS EM ADOLESCENTES DO MUNICÍPIO DE ALEGRE-ES
}

\author{
Veronica da Fonseca Almeida ${ }^{1}$ \\ Herbert de Paula ${ }^{2}$ \\ Luciane Daniele Cardoso ${ }^{3}$
}

Resumo: A síndrome metabólica (SM) é caracterizada por um conjunto de alterações metabólicas que incluem a intolerância à glicose, hipertensão arterial sistêmica, aumento dos triacilgliceróis e redução da lipoproteína de alta densidade (HDL). Objetivou-se avaliar a prevalência da SM e de fatores de risco cardiovascular (FRCV) entre adolescentes, bem como a relação destes com a resistência insulínica (RI). Trata-se de um estudo transversal no qual foram avaliados 293 adolescentes entre 11 e 15 anos. A prevalência de SM foi de 7,5\%. A HDL baixa foi o FRCV mais frequente, seguida de elevação do colesterol total e hipertrigliceriemia A resistência insulínica esteve presente em $14 \%$ dos adolescentes e apresentouse positivamente correlacionada, com o perímetro da cintura $(r=0,36)$, colesterol total $(r=0,046$,$) ,$ triacilgliceróis $(r=0,065)$ e negativamente com a lipoproteína de alta densidade $(r=-0,042)$. A prevalência elevada da SM e RI em adolescentes bem como a associação positiva entre RI e FRCV isolados sugere a origem precoce das alterações cardiovasculares justificando medidas interventivas capazes de reduzir a morbimortalidade na idade adulta.

Palavras-chave: Síndrome metabólica; Resistência insulínica; Adolescente; Fatores de risco cardiovascular.

\footnotetext{
${ }^{1}$ Ciências da Saúde e Epidemiologia. Universidade Federal do Espírito Santos-Campus Alegre/ES, Brasil. E-mail: veronica_fonseca18@hotmail.com.

${ }^{2}$ Ciências da Saúde e Epidemiologia. Universidade Federal do Espírito Santos-Campus Alegre/ES, Brasil. E-mail: hdpaula@gmail.com

${ }^{3}$ Ciências da Saúde e Epidemiologia. Universidade Federal do Espírito Santos-Campus Alegre/ES, Brasil. E-mail: Idcardosontr@gmail.com.
} 